\title{
Synthesis of Vinylene-Linked Covalent Organic Frameworks by Monomer-Self-Catalyzed Activation of Knoevenagel Condensation
}

\author{
Shuai $\mathrm{Bi}^{\S}$, Fancheng Meng§ , Dongqing Wu and Fan Zhang* \\ School of Chemistry and Chemical Engineering, State Key Laboratory of Metal Matrix Composites, Shanghai Jiao Tong University, \\ Shanghai 200240, China
}

\begin{abstract}
Reticular chemistry on the basis of thermodynamically controlled linking modes and numerous organic building blocks has constituted versatile crystalline frameworks in molecular-level precision. However, vinylene-linked organic frameworks (COFs) are still quite far from flexible tailoring either in their structures or topologies, due to the lack of monomers with sufficient activities. Herein, we established a strategy to synthesize vinylene-linked COFs via Knoevenagel condensation of a tetratopic monomer 2,2',6,6'-tetramethyl-4,4'-bipyridine (TMBP) with linear aromatic dialdehydes in a mixed solvent of benzoic anhydride and benzoic acid. Mechanism investigation suggested that the condensation was promoted by a pyridine-self-catalyzed benzoylation upon the cleavage of benzoic anhydride solvent molecules. The layered structures of the resultant COFs were highly crystallized into orthorhombic lattice with vertically aligned AA stacking mode, delivering high surface areas up to $1560 \mathrm{~m}^{2} \mathrm{~g}^{-1}$. The $\pi$-extended conjugated skeletons comprising para-bipyridyl units and vinylene linkages endow these COFs with substantial semiconducting properties, releasing visible light-stimulated catalytic activity in water-splitting hydrogen evolution with a rate as high as $3300 \mu \mathrm{mol}$ $\mathrm{g}^{-1} \mathrm{~h}^{-1}$.
\end{abstract}

\section{INTRODUCTION}

Covalent organic frameworks (COFs) represent an emerged class of high crystalline porous polymers with well-defined periodic structures, which not only allows one to rationally design and tailor their chemical structures or topologies, but also readily manipulate them in various applications on the basis of establishing the essential structure-property relationships and understanding the related mechanism ${ }^{1-3}$. Among them, vinylene-linked organic frameworks (COFs) feature prominent stability and $\pi$-electron communication, likely helpful for expanding the potential applications of COFs, in particular, in the field of semiconductor ${ }^{4-6}$. Thermodynamically controlled Knoevenagel condensation is one of the most powerful tools in the formation of carboncarbon double bond linkage ${ }^{7-10}$. Unlike those traditional dynamic covalent chemistry, such condensation involves the generation of active intermediates, such as, carbon anions, which is crucial for efficiently boosting chain growth and correcting defect through self-healing processes for a $\mathrm{COF}^{11-14}$. Typically, an available monomer bearing aryl $\alpha$-carbon atoms, has to be enough electron-deficient for enhancing the $\alpha$-proton acidity and stabilizing the reactive intermediate, commonly achieved by the introduction of multiple electron-withdrawing moieties, such as cyano group, pyridyl nitrogen ${ }^{15-16}$. However, these modifications normally means a tedious preparation and the utilization of toxic reagent (e.g. $\mathrm{NaCN}$ or $\mathrm{CuCN}$ ), meanwhile, significantly constrains the accessible reaction sites of a monomer, severely limiting its linking modes. Correspondingly, on the basis of very few monomers, vinylenelinked COFs with hexagonal crystal system occupied the major ratio, and several single-pore orthorhombic skeletons were originating from the ditopic monomer 1,4- phenylenediacetonitrile ${ }^{17}$. While, a monomer with more $\alpha$ carbon atoms $(\geqslant 4)$ suitable for Knoevenagel condensation could not be found so far.

Pyridyl nitrogen-containing molecules are widely used in catalysis, supramolecular chemistry, and organic semiconducting materials, associated with their intrinsic Lewis basicity, substantial physical properties, and self-assembling behaviors ${ }^{18}$. Knoevenagel reaction holds some merits in the preparation of vinylene-linked pyridine-based small molecules or linear conjugated polymers using methyl-substituted pyridine, due to low-cost, environmentally friendly reagents, less unidentified reactive intermediates, and only trans-alkene formation ${ }^{19-21}$. Unfortunately, the mechanism of such traditional reaction seems never to be investigated.

Typically, functionalization at nitrogen to generate pyridinium cations, makes pyridine ring favorable for electrophilic and/or nucleophilic substitution. Very recently, we reported a Knoevenagel condensation at the three methyl carbons in one pyridine promoted upon quaternization, leading to the formation of several pyridinium-cored vinylene-linked COFs with high crystallinities and quite large surface areas, suggesting an excellent efficiency in the activation of a multitopic monomer ${ }^{22} \mathrm{~N}$-acyl pyridinium has been found as a highly active intermediate formed in the process of pyridine-catalyzed hydrolysis of acid anhydride, or as one type of efficient acylation reagent from the treatment of pyridine with an acyl donor $^{23-24}$. With these reasons in our mind, our effort is to explore a much lower-cost, less-toxic and concise method to construct vinylene-linked COFs by virtue of methyl substituted pyridine-based monomer, and attempt to establish the optimized reaction conditions and corresponding thermodynamically controlled mechanism. 
Scheme 1. Synthesis and structures of (a) model molecule, (b) COF-DFB and COF-BPDA.

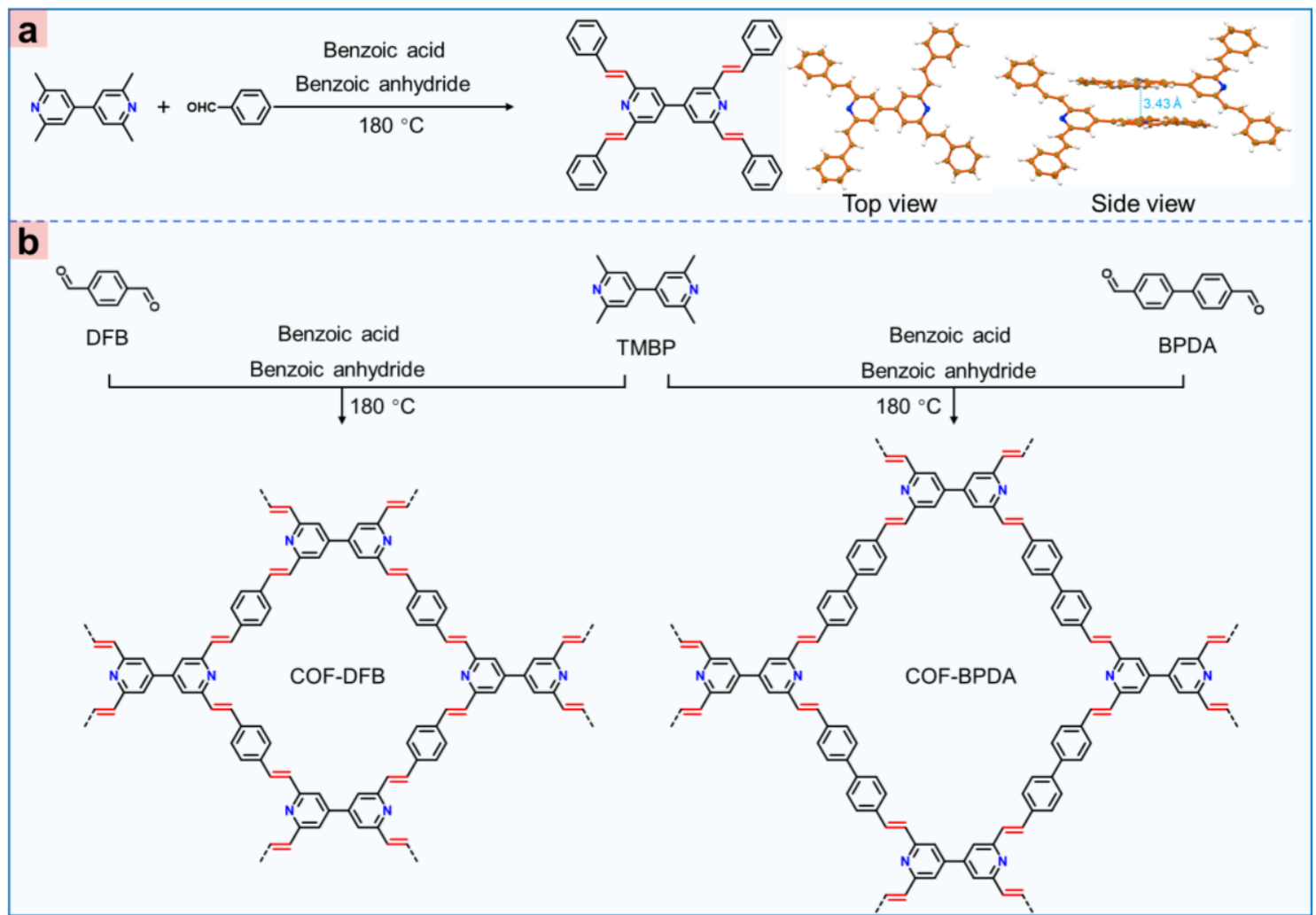

In this work, we present the preparation of two vinylene-linked COFs with orthorhombic reticular topology by condensation of

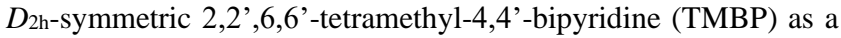
tetratopic monomer with the ditopic linear aromatic dialdehydes in benzoic anhydride and benzoic acid. The reaction mechanism manifested that the pyridine units in monomer TMBP enable catalyzing their benzoylation via the cleavage of benzoic anhydride solvent molecules, thus helpfully activating the aryl $\alpha$-carbon atoms for Knoevenagel reaction. The resulting COFs with high crystallinities and surface areas, were considerably characterized. Their $\pi$-extended conjugated skeletons endow them with excellent semiconducting properties and corresponding application, such as, visible light catalysis of water-splitting hydrogen evolution.

\section{RESULTS AND DISCUSSION}

Traditionally, in order to activate the methyl groups of pyridine ring for Knoevenagel condensation, incorporation of the electronwithdrawing substituents or quaternization at pyridine ring is necessary. Here, our attempt is to take use of the Lewis basicity of pyridine moiety for promoting the Knoevenagel reaction of a newly designed pyridine-based monomer 2,2',6,6'-tetramethyl-4,4'bipyridine (TMBP), bearing four pyridylmethyl terminal groups. Some previous works reported that methyl-substituted pyridine derivatives were facile to conduct Knoevenagel reaction with aromatic aldehydes in a mixture solvent of acetic anhydride and acetic acid at $c a .135{ }^{\circ} \mathrm{C}$ with moderate yield $(<60 \%)^{25}$. Considering the relatively weak reversibility of such carbon-carbon double bond formation reaction, we decided to raise the reaction temperature in a mixed solvent of benzoic anhydride and benzoic acid for the preparation of model compound and $\mathrm{COFs}^{26}$.

The key monomer TMBP was prepared via a facile one-step Yamamoto coupling in high yield ${ }^{27}$. The Knoevenagel condensation between TMBP and benzaldehyde was performed in a mixture of benzoic acid and benzoic anhydride under $180{ }^{\circ} \mathrm{C}$, affording the model molecule 2,2',6,6'-tetrastyryl-4,4'-bipyridine in a yield of $85 \%$ (synthesis detailed in the Supporting Information). The structures of these new compounds were unambiguously characterized by NMR, MALDI-TOF MS and single crystal X-ray diffraction analyses. The packing diagram of the model molecule showed a $\pi$-dimer arrangement of the neighbouring molecules with a distance of $3.43 \AA$, manifesting the distinct $\pi-\pi$ interaction. By using the condition of the model reaction, TMBP was copolymerized with 1,4-diformylbenzene (DFB) or 4,4biphenyldialdehyde (BPDA), respectively, to synthesize COFs. In a typical protocol, TMBP, DFB, benzoic anhydride and benzoic acid in a molar ratio of 1:2:2:0.2 was sealed in glass ampoule and heated at $180{ }^{\circ} \mathrm{C}$ for 3 days (Scheme 1), leading to the generation of large amount of red-brown precipitates. After washed with base solution and organic solvents (methanol, THF and DCM), the resultant crude product was converted to yellow powder in $90 \%$ isolated yield (termed COF-DFB). Similarly, another COF (COFBPDA) were derived from a larger aldehyde monomer (BPDA) as yellow powders in $94 \%$ yield.

It is well-known that, acylation or alkylation at the pyridyl nitrogen always leads to nucleophilic attack on the pyridine ring due to the strengthened electron deficiency ${ }^{18,28}$. Alkylation at the pyridyl nitrogen has been verified to efficiently promote a Knoevenagel condensation at the pyridylmethyl carbon atoms ${ }^{22,29}$. Considering that pyridine can act as Lewis base for catalyzing hydrolysis of acetic anhydride through the formation of the $\mathrm{N}$ acetyl pyridinium ion intermediate ${ }^{23}$, under our circumstance, the proposed mechanism could begin with the benzoylation of methylpyridine moieties by benzoic anhydride to give the benzoyl pyridinium salt. The electron-deficient character of pyridine ring was significantly increased by the benzoylation, thus favorable for the enhancement of $\mathrm{C}-\mathrm{H}$ acidity on the pyridyl methyl. Meanwhile, the generated conjugated base benzoate also could capture the protons at the methyl groups, leading to the formation of enamine intermediate. Subsequently, electrophilic attack of the protonated aldehyde in the presence of benzoic acid, to the above 
Scheme 2. Proposed Mechanism for Benzoic AnhydrideCatalyzed Knoevenagel Condensation of Methylpyridine and Benzaldehyde via Acyl Pyridinium Intermediate.

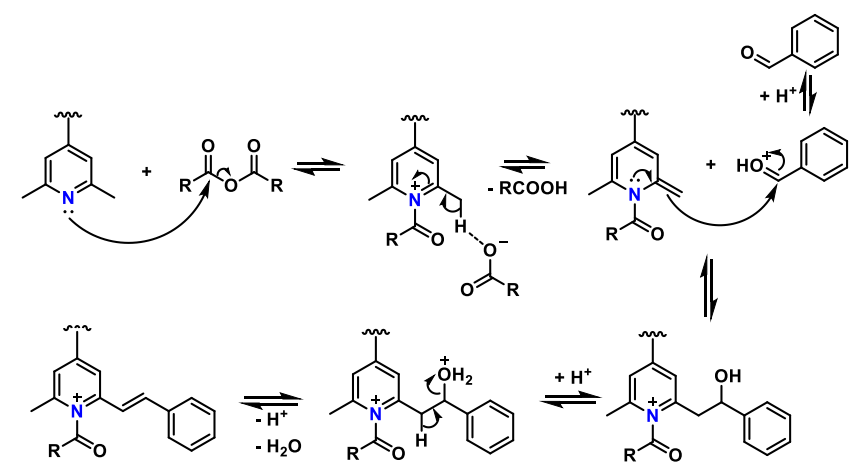

enamine intermediates, followed by the elimination of molecular water, affords the carbon-carbon double bond linkages. For further illustration, several controlled experiments were conducted. First, the formation of the key intermediate benzoyl pyridinium moiety in a reaction of benzoic anhydride and trimethylpyridine was confirmed by GC-MS analysis, somehow, supporting the selfcatalyzed cleavage of benzoic anhydride by the pyridine-based monomer during the preparation of COFs (Figure S1, detailed see the Supporting Information). Additionally, the aforementioned model molecule 2,2',6,6'-tetrastyryl-4,4'-bipyridine could be efficiently achieved by the reaction of TMBP and benzaldehyde in a mixture of benzoic chloride as acylation reagent and sodium benzoate as conjugated base, indicative of the acylation-activated Knoevenagel condensation. Notably, similar to those previous reports, such intermediate benzoyl pyridinium is quite unstable, which readily recovered to pyridine even in the presence of trace water.
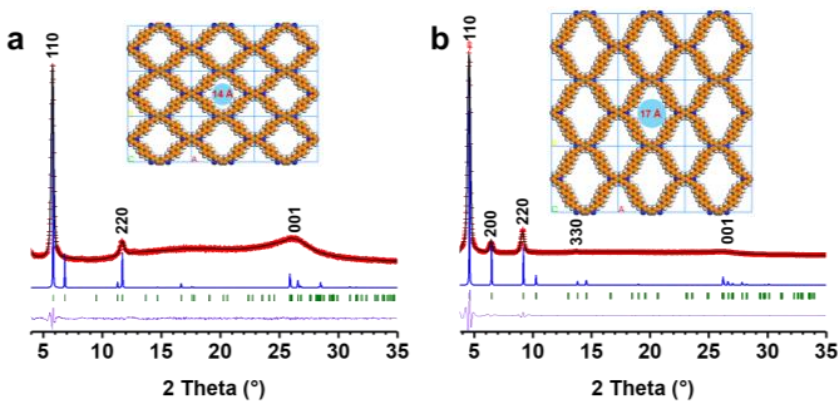

Figure 1. PXRD patterns of (a) COF-DFB and (b) COF-BPDA. Comparison between the experimental profiles (red cross), Pawley refined profiles (black line), the simulated patterns for eclipsed (AA) stacking mode (blue line), the Bragg positions (green bar) and the refinement differences (violet line). Inset: Corresponding refined 2D crystal structural models of COF-DFB and COF-BPDA assuming the eclipsed (AA) stacking mode.

The crystallinity of COF-DFB was examined by powder X-ray diffraction (PXRD) measurements. Theoretically, the condensation of a $D_{2 \mathrm{~h}}$ monomer and a $C_{2}$ monomer can give rise to two possible 2D COF structures, that is, an orthorhombic system that bears only a single-pore framework and a hexagonal system that possesses two different kinds of pores with Kagome lattice ${ }^{30}$. Indexing of the PXRD pattern of COF-DFB identified the space group Cmmm (No. $65)$, indicating an orthorhombic lattice. The diffraction peaks at $5.81^{\circ}, 11.67^{\circ}$ and $25.90^{\circ}$ could be assigned to (110), (220) and (001) facets, respectively (Figure 1a). The experimental PXRD pattern of
COF-DFB was in accordance with simulated pattern of a orthorhombic lattice with AA stacking mode, whereas the staggered $\mathrm{AB}$ stacking model and Kagome lattices exhibit significant difference from the experimental PXRD data (Figures S21 and S22). The first dominant diffraction peak at $5.81^{\circ}$ corresponds to the $d$-spacing of $1.52 \mathrm{~nm}$, which was in good agreement with the calculated diameter of the rhombic pore (1.4 $\mathrm{nm}$, Figure S18). Furthermore, the profile of Pawley refinement matched well with the observed signals as evident by the residual $R_{\mathrm{wp}}$ and $R_{\mathrm{p}}$ values converged to $3.79 \%$ and $2.94 \%$, respectively, affording unit cell parameters of $a=25.872 \AA, b=18.625 \AA, c=$ $3.439 \AA, \alpha=\beta=\gamma=90^{\circ}$.

Similarly, COF-BPDA exhibits diffraction peaks at $4.59^{\circ}, 6.45^{\circ}$, $9.15^{\circ}, 13.76^{\circ}$, and $26.21^{\circ}$, which can be assigned to the (110), (200), (220), (330) and (001) facets, respectively (Figure 1b). It can be found that the simulated orthorhombic lattice with AA stacking mode well reproduced the experimentally observed PXRD pattern in terms of peak positions and relative intensities. The unit cell parameters were refined by the Pawley method using PXRD data $\left(a=b=27.274 \AA, c=3.398 \AA, \alpha=\beta=\gamma=90^{\circ}\right)$ with residual factors of $R_{\mathrm{wp}}=8.74 \%$ and $R_{\mathrm{p}}=6.40 \%$.
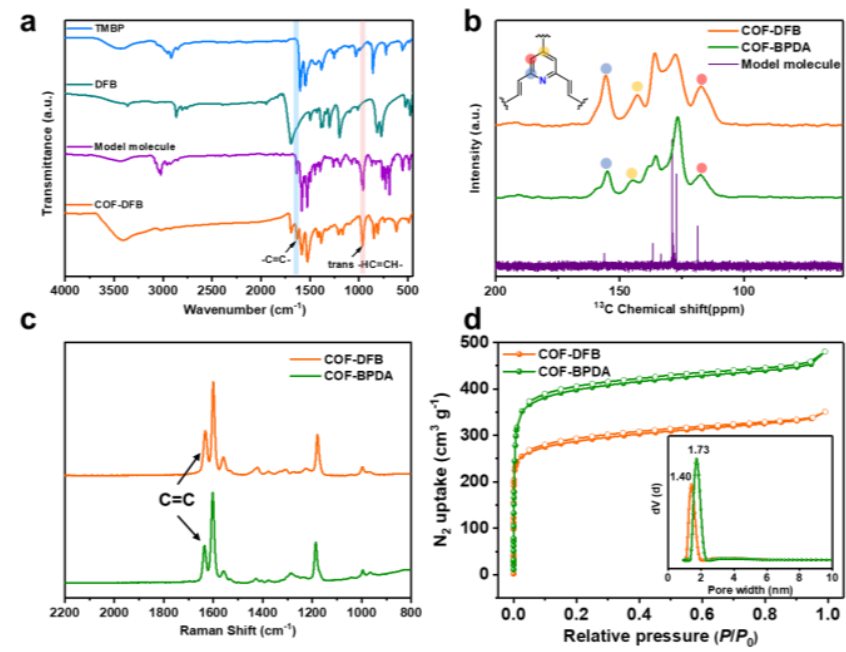

Figure 2. (a) FT-IR spectra of model molecule, COF-DFB and corresponding monomers. (b) ${ }^{13} \mathrm{C}$ NMR spectra of COF-DFB, COF-BPDA and model molecule. (c) Raman spectra of COF-DFB and COF-BPDA. (d) Nitrogen sorption isotherms of COF-DFB and COF-BPDA. Inset: Pore size distribution calculated from quenched solid density functional theory.

Fourier transform infrared (FT-IR) spectra of COF-DFB and COF-BPDA were analyzed by comparison with the monomer and the model molecule (Figure 2a and Figure S2). The disappearance of $\mathrm{C}-\mathrm{H}$ vibration $\left(2916 \mathrm{~cm}^{-1}\right)$, attenuation of $\mathrm{C}=\mathrm{O}$ stretching band $\left(1694 \mathrm{~cm}^{-1}\right)$ and appearance of new intensive vinyl $(\mathrm{C}=\mathrm{C})$ bands at 961 and $1631 \mathrm{~cm}^{-1}$, confirmed the high polycondensation degree of COFs. These observations were further confirmed by the complementary Raman spectra analysis (Figure 2c), which showed the characteristic Raman signals at $1630 \mathrm{~cm}^{-1}$ for the $\mathrm{C}=\mathrm{C}$ stretch vibration. The formation of vinyl-linked COFs was further verified by ${ }^{13} \mathrm{C}$ cross-polarization magic angle spinning (CP-MAS) solidstate NMR spectroscopy, showing good match with the ${ }^{13} \mathrm{C}$ NMR spectra of the model molecule (Figure $2 b$ and Figure S3). The permanent porosity of COF-DFB and COF-BPDA was assessed by $\mathrm{N}_{2}$ sorption measurements at $77 \mathrm{~K}$ (Figure $2 \mathrm{~d}$ ). Both COFs show the reversible type I isotherms with rapid $\mathrm{N}_{2}$ uptakes at the low relative pressure range $P / P_{0}<0.05$, characteristic of microporous materials. Brunauer-Emmett-Teller (BET) surface areas calculated 
from $\mathrm{N}_{2}$ adsorption isotherms revealed that the COF-DFB and COF-BPDA exhibited high specific surface areas of $1104 \mathrm{~m}^{2} \mathrm{~g}^{-1}$ and $1560 \mathrm{~m}^{2} \mathrm{~g}^{-1}$, respectively. The pore size distribution analysis of COF-DFB and COF-BPDA based on the quenched solid density functional theory (QSDFT) indicates one main distribution centered at $1.40 \mathrm{~nm}$ and $1.70 \mathrm{~nm}$, respectively, which is in consistent with the pore diameter based on the Conolly surface in their eclipsed (AA) layer stacking mode (Figures S18 and S19).
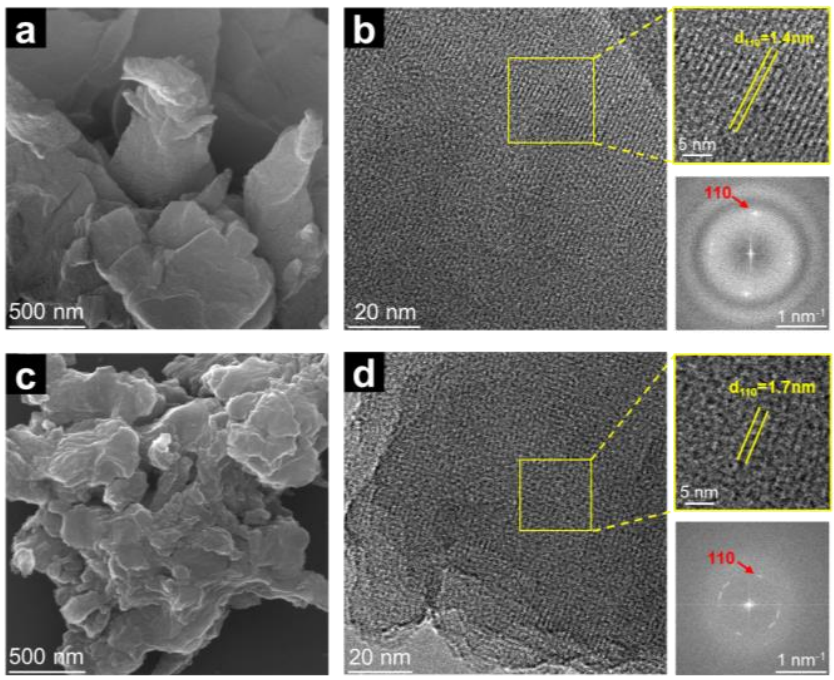

Figure 3. SEM images of (a) COF-DFB and (c) COF-BPDA. HRTEM images of (b) COF-DFB and (d) COF-BPDA. Upper right: enlarged view of a selected area indicating the distance of 110 plane. Low right: fast Fourier transform (FFT) pattern.

The morphologies and microstructures of the two COFs were studied by scanning electron microscopy (SEM) and Highresolution transmission electron microscopy (HRTEM). The SEM images show that COF-DFB and COF-BPDA are composed of layered blocks (Figures $3 \mathrm{a}$ and $3 \mathrm{c}$ ). HRTEM images exhibit longrange ordered microstructures of the two COFs. The lattice fringes was clearly visualized in Figures $3 b$ and $3 d$, suggesting the high crystallinities of these samples. One-dimensional channels with uniform distance of $1.40 \mathrm{~nm}$ and $1.70 \mathrm{~nm}$ for COF-DFB and COFBPDA was clearly visualized along the [001] direction, corresponding to the $d$-spacing of (110) facet. Fast Fourier transform (FFT) of the raw HRTEM images generates a tetragonal arrangement of four white diffraction spots, corresponding to (110) facet (low right of Figures $3 b$ and $3 d$ ). A rhombic porous structure can be observed on the HRTEM image of COF-BPDA, further indicating the formation of orthorhombic lattice, which is in accordance with the PXRD analysis (Figure S7).

The electronic structures of the two COFs were systematically investigated by ultraviolet/visible diffuse reflectance spectroscopy (UV/vis DRS), photoluminescence (PL) spectroscopy and ultraviolet photoelectron spectroscopy (UPS). As shown in Figure $4 \mathrm{a}$, COF-DFB shows an absorption maxima at $c a .475 \mathrm{~nm}$, and COF-BPDA exhibits a relatively broad absorption band with a tail covering $450 \sim 700 \mathrm{~nm}$. Accordingly, the optical band gaps of the COFs were determined from Kubelka-Munk function as $2.5 \mathrm{eV}$ and $2.3 \mathrm{eV}$ for COF-DFB and COF-BPDA, respectively (Figure $4 \mathrm{a}$ inset). The energy of valence band maximum $\left(E_{\mathrm{VB}}\right)$ of the three COFs relative to vacuum level were evaluated by UPS analyses (Figure S9). The EvB values of COF-DFB and COF-BPDA were calculated by subtracting the UPS width from excitation energy (He I, $21.2 \mathrm{eV}$ ), which were -6.4 and $-6.3 \mathrm{eV}$, respectively. Coupled with their optical band gaps, the energy of conduction band

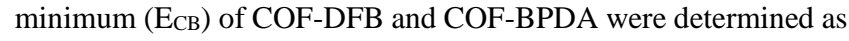
-3.9 and $-4.0 \mathrm{eV}$, respectively. The $\mathrm{E}_{\mathrm{CB}}$ of both $\mathrm{COFs}$ are positioned higher than the reduction potential of proton $\left(\mathrm{E}_{\mathrm{red}} \mathrm{H}^{+} / \frac{\mathbf{1}}{\mathbf{2}} \mathrm{H}_{2}\right.$ $=-4.44 \mathrm{eV}$ ) (Figure 4c), allowing for reducing proton to hydrogen once the COFs were excited by light with photon energy larger than their band gaps. The steady-state photoluminescence (PL) spectra of the two COFs were measured. Upon excitation at $365 \mathrm{~nm}$, the emission maxima of COF-BPDA appeared at $\lambda_{\mathrm{em}}=605 \mathrm{~nm}$, distinctly red-shifted by $30 \mathrm{~nm}$ over that of $\lambda_{\mathrm{em}}=575 \mathrm{~nm}$ for COFDFB (Figure $4 \mathrm{~b}$ ), attributable to the larger $\pi$-extended structure of COF-BPDA. Additionally, the PL decay curves of two COFs are well fitted with the lifetimes with two exponential components (Figure S9). The average lifetimes of COF-DFB and COF-BPDA were estimated to be 7.08 and $7.56 \mathrm{~ns}$, respectively. The longer lifetime of COF-BPDA indicates the suppressed radiative recombination of photogenerated excitons, associated with its extended $\pi$-conjugated backbone.
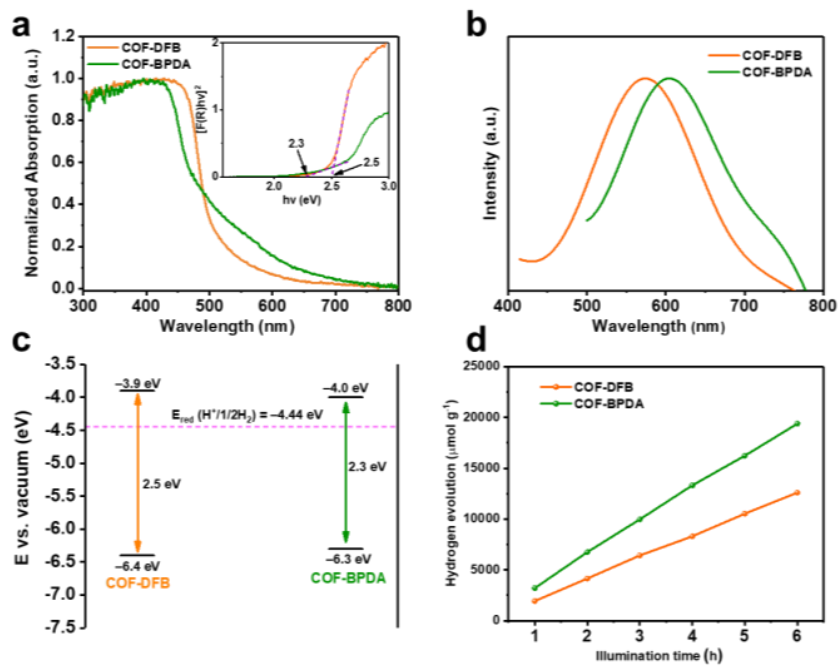

Figure 4. (a) UV/vis DRS of COF-DFB and COF-BPDA. Inset: the band gaps determined from Kubelka-Munk-transformed reflectance spectra. (b) Steady-state photoluminescence (PL) spectra of COF-DFB and COF-BPDA. (c) Band structure of COFDFB and COF-BPDA. (d) Time-course photocatalytic $\mathrm{H}_{2}$ production under visible light $(\lambda>420 \mathrm{~nm})$ for COF-DFB and COF-BPDA (20.0 mg sample in $100 \mathrm{~mL}$ water, $3 \mathrm{wt} \% \mathrm{Pt}, 0.1 \mathrm{M}$ ascorbic acid).

As a proof of concept, we studied the visible light photocatalytic $\mathrm{H}_{2}$ evolution activities of COF-DFB and COF-BPDA from water reduction. Both $\mathrm{COF}$ samples can be easily dispersed in water, which is related to their pyridine-rich backbones that strongly interacted with water. The photocatalysis experiments were performed by dispersing $20.0 \mathrm{mg}$ of COF powder into $0.1 \mathrm{M}$ aqueous ascorbic acid solution, in which ascorbic acid was applied as a sacrificial electron donor, and $3 \mathrm{wt} \%$ platinum was in situ photo-deposited on the COF. Upon visible light illumination, COFBPDA showed an average hydrogen evolution rate (HER) of $3229.01 \mu \mathrm{mol} \mathrm{h}^{-1} \mathrm{~g}^{-1}$, nearly 1.54 times faster than that of COFDFB $\left(2099.60 \mu \mathrm{mol} \mathrm{h}^{-1} \mathrm{~g}^{-1}\right)$ (Figure 4d). This indicated that the photocatalytic $\mathrm{H}_{2}$ generation performance of COF-DFB and COFBPDA were mainly influenced by their intrinsic semiconducting properties. COF-BPDA with longer conjugation length and narrower band gap delivered higher activity as compared to COFDFB.

\section{CONCLUSIONS}


In conclusion, we have established an efficient synthetic strategy that can combine a $D_{2 \mathrm{~h}}$ symmetrical building block 2,2',6,6'tetramethyl-4,4'-bipyridine with $C_{2}$ symmetrical aromatic dialdehyde linkers to construct vinylene-linked COFs through Knoevenagel condensation in a mixed solvent of benzoic anhydride/benzoic acid. The possible reaction mechanism involves the self-catalyzed benzoylation of the used pyridine-based monomer, which subsequently promoted the Knoevenagel condensation at the pyridylmethyl carbon atoms of this monomer. The as-synthesized COFs were highly crystallized in orthorhombic lattices, releasing relatively large surface areas. The fully $\pi$ conjugated structures give rise to abundant semiconducting properties of the COFs, allowing for efficiently visible lightcatalyzed hydrogen evolution from water reduction. This strategy paves a low-cost, environmentally friendly pathway to enlarge the regimes of vinylene-linked COFs with complex geometric and topological structures, as well as intriguing properties.

\section{EXPERIMENTAL SECTION}

All experimental procedures are provided in the Supporting Information.

\section{ASSOCIATED CONTENT}

\section{Supporting Information.}

The Supporting Information is available free of charge on the ACS Publications website at http://pubs.acs.org. Detailed synthetic procedures, characterization of materials and photocatalytic experiments.

\section{Corresponding Author}

*fan-zhang@sjtu.edu.cn

\section{Author Contributions}

${ }^{\S}$ S. B. and F. M. contributed equally.

\section{Notes}

The authors declare no competing financial interest.

\section{ACKNOWLEDGMENT}

This work was supported by the Shanghai Committee of Science and Technology (20JC1414900), the National Natural Science Foundation of China $(22005189,21774072,22075178)$, and the China Postdoctoral Science Foundation (2020M681277).

\section{REFERENCES}

1. Côté, A. P.; Benin, A. I.; Ockwig, N. W.; O'Keeffe, M.; Matzger, A. J.; Yaghi, O. M., Porous, Crystalline, Covalent Organic Frameworks. Science 2005, 310 (5751), 1166-1170.

2. Diercks, C. S.; Yaghi, O. M., The atom, the molecule, and the covalent organic framework. Science 2017, 355 (6328), eaal1585.

3. Li, Y.; Chen, W.; Xing, G.; Jiang, D.; Chen, L., New synthetic strategies toward covalent organic frameworks. Chem. Soc. Rev. 2020, 49 (10), 2852-2868.

4. Bi, S.; Yang, C.; Zhang, W.; Xu, J.; Liu, L.; Wu, D.; Wang, X.; Han, Y.; Liang, Q.; Zhang, F., Two-dimensional semiconducting covalent organic frameworks via condensation at arylmethyl carbon atoms. Nat. Commun. 2019, 10 (1), 2467.

5. Lyu, H.; Diercks, C. S.; Zhu, C.; Yaghi, O. M., Porous Crystalline Olefin-Linked Covalent Organic Frameworks. J. Am. Chem. Soc. 2019, 141 (17), 6848-6852.

6. Jadhav, T.; Fang, Y.; Patterson, W.; Liu, C.-H.; Hamzehpoor, E.; Perepichka, D. F., 2D Poly(arylene vinylene) Covalent Organic Frameworks via Aldol Condensation of Trimethyltriazine. Angew. Chem., Int. Ed. 2019, 58 (39), 13753-13757.
7. Zhuang, X.; Zhao, W.; Zhang, F.; Cao, Y.; Liu, F.; Bi, S.; Feng, $\mathrm{X}$., A two-dimensional conjugated polymer framework with fully sp2bonded carbon skeleton. Polym. Chem. 2016, 7 (25), 4176-4181.

8. Jin, E.; Asada, M.; Xu, Q.; Dalapati, S.; Addicoat, M. A.; Brady, M. A.; Xu, H.; Nakamura, T.; Heine, T.; Chen, Q.; Jiang, D., Twodimensional $\mathrm{sp} 2$ carbon-conjugated covalent organic frameworks. Science 2017, 357 (6352), 673-676.

9. Gu, R.; Flidrova, K.; Lehn, J.-M., Dynamic Covalent Metathesis in the $\mathrm{C}=\mathrm{C} / \mathrm{C}=\mathrm{N}$ Exchange between Knoevenagel Compounds and Imines. J. Am. Chem. Soc. 2018, 140 (16), 5560-5568.

10. König, N. F.; Mutruc, D.; Hecht, S., Accelerated Discovery of $\alpha$ Cyanodiarylethene Photoswitches. J. Am. Chem. Soc. 2021, 143 (24), 9162-9168.

11. Knoevenagel, E., 1,5-Diketone. Justus Liebigs Ann. Chem. 1894, $281,25-126$.

12. Hann, A. C. O.; Lapworth, A., VII.- Optically active esters of $\beta$ ketonic and $\beta$-aldehydic acids. Part IV. Condensation of aldehydes with menthyl acetoacetate. J. Chem. Soc., Trans. 1904, 85 (0), 46-56.

13. Cope, A. C., Condensation Reactions. I. The Condensation of Ketones with Cyanoacetic Esters and the Mechanism of the Knoevenagel Reaction. J. Am. Chem. Soc. 1937, 59 (11), 2327-2330.

14. Bi, S.; Thiruvengadam, P.; Wei, S.; Zhang, W.; Zhang, F.; Gao, L.; Xu, J.; Wu, D.; Chen, J.-S.; Zhang, F., Vinylene-Bridged TwoDimensional Covalent Organic Frameworks via Knoevenagel Condensation of Tricyanomesitylene. J. Am. Chem. Soc. 2020, 142 (27), 11893-11900.

15. Bi, S.; Lan, Z. A.; Paasch, S.; Zhang, W.; He, Y.; Zhang, C.; Liu, F.; Wu, D.; Zhuang, X.; Brunner, E.; Wang, X.; Zhang, F., Substantial Cyano-Substituted Fully sp 2 -Carbon-Linked Framework: Metal-Free Approach and Visible-Light-Driven Hydrogen Evolution. Adv. Funct. Mater. 2017, 27 (39).

16. Xu, J.; He, Y.; Bi, S.; Wang, M.; Yang, P.; Wu, D.; Wang, J.; Zhang, F., An Olefin-Linked Covalent Organic Framework as a Flexible Thin-Film Electrode for a High-Performance MicroSupercapacitor. Angew. Chem., Int. Ed. 2019, 58 (35), 12065-12069.

17. Jin, E.; Li, J.; Geng, K.; Jiang, Q.; Xu, H.; Xu, Q.; Jiang, D., Designed synthesis of stable light-emitting two-dimensional $\mathrm{sp} 2$ carbon-conjugated covalent organic frameworks. Nat. Commun. 2018, $9(1), 4143$

18. Bull, J. A.; Mousseau, J. J.; Pelletier, G.; Charette, A. B., Synthesis of Pyridine and Dihydropyridine Derivatives by Regio- and Stereoselective Addition to N-Activated Pyridines. Chem. Rev. 2012, 112 (5), 2642-2713.

19. Attias, A.-J.; Cavalli, C.; Bloch, B.; Guillou, N.; Noël, C., New Liquid Crystalline Conjugated Derivatives of 3,3'-Bipyridine as Components for Optoelectronic Materials. Chem. Mater. 1999, 11 (8), 2057-2068.

20. Attias, A.-J.; Hapiot, P.; Wintgens, V.; Valat, P., Tuning of the Mesogenic, Electronic, and Optical Properties of New Conjugated 3,3 ${ }^{6}$ Bipyridine Derivatives. Chem. Mater. 2000, 12 (2), 461-471.

21. Lemaître, N.; Attias, A.-J.; Ledoux, I.; Zyss, J., New SecondOrder NLO Chromophores Based on 3,3'-Bipyridine: Tuning of Liquid Crystal and NLO Properties. Chem. Mater. 2001, 13 (4), 1420-1427.

22. Meng, F.; Bi, S.; Sun, Z.; Jiang, B.; Wu, D.; Chen, J.-S.; Zhang, F., Synthesis of Ionic Vinylene-Linked Covalent Organic Frameworks through Quaternization-Activated Knoevenagel Condensation. Angew. Chem., Int. Ed. 2021, 60 (24), 13614-13620.

23. Fersht, A. R.; Jencks, W. P., Acetylpyridinium ion intermediate in pyridine-catalyzed hydrolysis and acyl transfer reactions of acetic anhydride. Observation, kinetics, structure-reactivity correlations, and effects of concentrated salt solutions. J. Am. Chem. Soc. 1970, 92 (18), 5432-5442.

24. Yanagi, M.; Imayoshi, A.; Ueda, Y.; Furuta, T.; Kawabata, T., Carboxylate Anions Accelerate Pyrrolidinopyridine (PPy)-Catalyzed 
Acylation: Catalytic Site-Selective Acylation of a Carbohydrate by in Situ Counteranion Exchange. Org. Lett. 2017, 19 (12), 3099-3102.

25. Bhowmik, P. K.; Nedeltchev, A. K.; Han, H., Synthesis, optical, and thermal properties of conjugated, bispyridyl and tetrapyridyl compounds by Knoevenagel reaction. Tetrahedron Lett. 2007, 48 (31), 5383-5387.

26. Wang, Z.; Yang, Y.; Zhao, Z.; Zhang, P.; Zhang, Y.; Liu, J.; Ma, S.; Cheng, P.; Chen, Y.; Zhang, Z., Green synthesis of olefin-linked covalent organic frameworks for hydrogen fuel cell applications. Nat. Commun. 2021, 12 (1), 1982.

27. Yoshizawa, M.; Nakagawa, J.; Kumazawa, K.; Nagao, M.; Kawano, M.; Ozeki, T.; Fujita, M., Discrete Stacking of Large
Aromatic Molecules within Organic-Pillared Coordination Cages. Angew. Chem., Int. Ed. 2005, 44 (12), 1810-1813.

28. Comins, D. L.; Abdullah, A. H., Regioselective addition of Grignard reagents to 1-acylpyridinium salts. A convenient method for the synthesis of 4-alkyl(aryl)pyridines. J. Org. Chem. 1982, 47 (22), 4315-4319.

29. Xu, X.; Qiu, W.; Zhou, Q.; Tang, J.; Yang, F.; Sun, Z.; Audebert, P., Nonlinear Optical Absorption Properties of Two Multisubstituted p-Dimethylaminophenylethenyl Pyridiniums. J. Phys. Chem. B 2008, 112 (16), 4913-4917.

30. Tian, Y.; Xu, S.-Q.; Liang, R.-R.; Qian, C.; Jiang, G.-F.; Zhao, $\mathrm{X}$., Construction of two heteropore covalent organic frameworks with Kagome lattices. CrystEngComm 2017, 19 (33), 4877-4881.

\section{Table of Content}

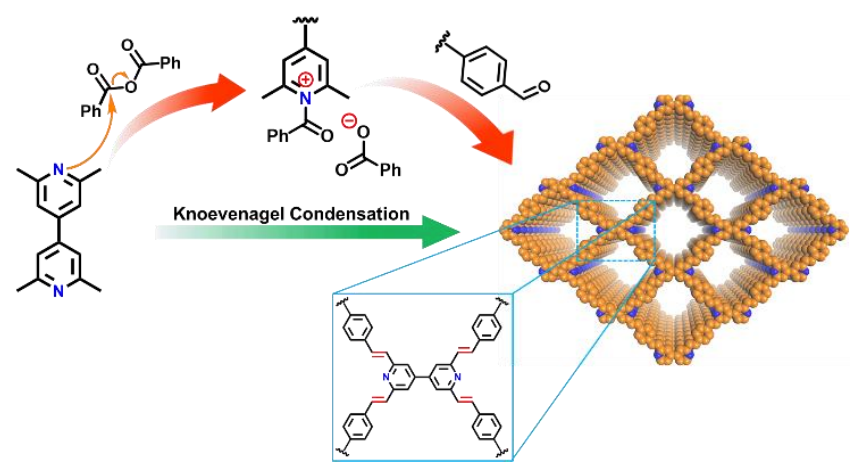

